\title{
Spatio-Temporal Data Model for Wireless Sensor Network
}

\author{
Kamel Abbassi ${ }^{1, *}$, Kamel khedhiri $^{2}$, Tahar Ezzedine ${ }^{1}$, Adnen Cherif ${ }^{2}$ \\ ${ }^{1}$ Communication System Laboratory Sys 'Com, National Engineering School of Tunis, University Tunis El Manar, BP \\ 37, Belvedere, 1002 Tunis \\ ${ }^{2}$ ATSSEE Research Laboratory, Science Faculty of Tunis, University Tunis El Manar, BP 37, Belvedere, 1002 Tunis, \\ Tunisia. \\ Corresponding author: kamel.abbassi@enit.utm.tn
}

This work is part of a Tunisian-South African cooperation scientific research project: Grant Numbers: 113340, 120106

\begin{abstract}
The monitoring system based on a sensor network requires large databases due to the storage of measurements without checking their redundancies. In this paper, we propose an instantaneous spatiotemporal data model based on object-oriented concepts that considers the possibility of tracking data from different objects (sensors) for periodic or instantaneous events. Each sensor must record its measurement only when a significant change is applied to it. With our model, we have subdivided the save sets into several sub entities to minimize the space occupied and save only the relevant information. This approach allows to manage the saving of different data according to the needs of the user or the phenomenon to be monitored. It also reduces data redundancy and facilitates the execution of queries.
\end{abstract}

Keywords: Wireless Sensor Network (WSN), Data Model, Snapshot, Geographic Information System (GIS), GeoUML

\section{INTRODUCTION}

The management of Spatio-Temporal data coming from the sensor network has been a topic of interest for many years. Indeed, the considered data can be evolutionary; it is interesting in this case to update the database or analyze the changes.

Different models of Spatio-Temporal databases exist. These models make it possible to store and manipulate Spatio-Temporal data. It consists of defining a Spatial-Temporal data model and implementing a basic conceptual model of spacetime data linked to the WSN.

To implement observations made on a phenomenon of any nature within a database, it is necessary to go through a preliminary step which is the deployment of a Spatio-Temporal data model and then conceptual modeling.

A model can be defined as "a formal representation of a phenomenon of the real world to a certain degree of approximation”.
In a database, the model will aim at graphically representing all the data and the interactions between them. The database is Spatio-Temporal in nature. It is interesting to think about an objectoriented model to highlight the various processes and scenarios of data variation in space and time aiming at monitoring the evolution of the different phenomena in the surveillance area to different entities, whether it is an event, periodic or instantaneous. To better understand the different characteristics of RTSTD (Real-Time SpatioTemporal Data), we propose a conceptual model of the specific data in GeoUML to evaluate the relationship between Spatio-Temporal data (ST) and Real-Time data (RT). However, this space-time GIS data model is capable of processing data types other than polygons such as points, lines, and responses to different types of spatial-temporal queries.

This paper is structured as follows: Section (2) is devoted to the presentation of the Spatio-Temporal 
data model. Section (3) discusses the proposed data model. The Spatio-Temporal Conceptual Model is represented in Section (4) as well as the description of the proposed Model. Section (5) processes the different queries on the data model. Section (6) discusses the results. Finally, Section (7) concludes this paper.

\section{SPATIO-TEMPORAL DATA MODEL}

The presentation of a spatial-temporal data model will use different methodologies depending on what is sought to be represented according to the studied phenomenon and the available data. Various solutions can be thus envisaged. Multiple searches have made considerable efforts to design new spatial data models such as the snapshot model [1], which is a class-oriented model that allows recording the state of a certain study area at a specific time.

Otherwise, the snapshot model can be eventbased or periodic. Another event-based model [2] records the study area when a specific event occurs. It is based on snapshot concepts [1] and the grid model [3]. The latter applies to the raster data and not to the vector data, the object-oriented model [4] is based on events. It consists of two-dimensional spatial objects and a third temporal dimension, which is associated with each object.

In this model, the record is associated with objects change in space and time. Objects that have nonspatial attribute changes are not registered and the three-domain models [5] oriented-class approach is composed of the semantic, temporal, and spatial domains, as well as domain links. Nevertheless, existing models present weaknesses such as data redundancy, implementation difficulties, and tracking of changes between two instants (attribute or spatial).

\section{PROPOSED DATA MODEL}

The reason why we construct the data model "Fig.1" is to develop predictions from a set of data representing a phenomenon. The model we propose is based on numerous studies carried out in the field of GIS [1] [3] [4] [6]. However, it broadens the temporal data model results [7]. This model allows passing from a so-called "oriented-territorial" approach to a so-called "Object-oriented" approach.

In this approach, the identity information of the object remains coherent in time; it is used to follow the spatial changes and attribute over time. It supports the recording of non-spatial and spatial attribute changes. These changes can be triggered by event-driven, periodic, or instantaneous. It integrates the modeling of events having an impact on the surveillance zone; that is to say, those, which cause modifications around the objects making up the environment.

Here, we propose a method for tracking phenomena by varying the attribute and spatial data over time of the various objects at the core of the model as well as a method of updating and maintaining this model. The recording time is the valid time; it allows representing the real-time detection of the measured value in the real mode. The attribute updates are represented according to the tuple-versioning method. We can also criticize the fact that all the objects will not be characterized by the same number of updates, which will make less complex the resolution of the Spatio-Temporal queries.

This solution offers two advantages compared to the "traditional snapshots" method that we have exposed: it first limits the redundancy of information since the update only affects the modified objects. Moreover, it makes it possible to manage quite easily the historicity of an object for the various updates carried out throughout its existence (from the reference period T0 to the present time Tn). In addition, a method for interactive analysis of these changes is proposed via a sensors network to provide the risk specialists with a tool that enables them to carry out expertise on events (sometimes on very old data or recent data) in real-time, which have affected the territory by confronting various sources. These appraisals, called Syntheses, will be used by risk managers to refine and complete their decision-making by referring to the dimension of the collected measures as well as the geographical location of the events.

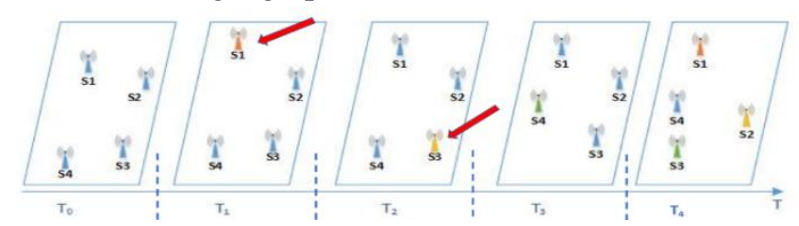

FIG.1: Snapshot model based on object-oriented concepts 
Fig.1 shows 4 types of sensor changes over time. At the beginning (T0) all the sensors in their locations.

At $\mathrm{T} 1$ the sensor $\mathrm{S} 1$ has been moved and change her value but the other sensors keep their locations.

At T2 all the sensors keep their location and the only difference is the new measurement recorded by the sensor S3.

At T3 all the cursors are moved and at the same time record new measures.

The following 4 tables show in detail these changes:

TABLE. 1: The initial state of implementation of the sensor network in the monitoring zone is carried out at $\mathbf{T}_{\mathbf{0}}$

\begin{tabular}{lccc}
\hline \hline Sensor & latitude & Longitude & value \\
\hline S1: Temperature & 36.830 & 10.146 & $21^{\circ} \mathrm{C}$ \\
S2: Humidity & 36.000 & 10.147 & $65 \%$ \\
S3: Sunshine & 36.920 & 10.650 & $700 \mathrm{w} / \mathrm{m}^{2}$ \\
S4: Wind speed & 36.500 & 10.710 & $12 \mathrm{Km} / \mathrm{h}$ \\
\hline \hline
\end{tabular}

TABLE. 2: The following table gives the recording of the spatial data during the change of position of the sensor $\mathrm{S} 1$ between $\mathbf{T}_{\mathbf{0}}$ and $\mathbf{T}_{\mathbf{1}}$

\begin{tabular}{llll}
\hline \hline Sensor & latitude & Longitude & value \\
\hline S1: Temperature & $\mathbf{3 6 . 4 3 0}$ & $\mathbf{1 0 . 1 2 0}$ & $21{ }^{\circ} \mathrm{C}$ \\
S2: Humidity & 36.000 & 10.147 & $65 \%$ \\
S3: Sunshine & 36.920 & 10.650 & $700 \mathrm{w} / \mathrm{m}^{2}$ \\
S4: Wind speed & 36.500 & 10.710 & $12 \mathrm{Km} / \mathrm{h}$ \\
\hline \hline
\end{tabular}

TABLE 3: The following table gives the recording of the attribute data upon detection of a change in value measured by the sensor $S_{3}$ between $T_{1}$ and $T_{2}$

\begin{tabular}{lccc}
\hline \hline \multicolumn{1}{c}{ Sensor } & latitude & Longitude & value \\
\hline $\mathrm{S}_{1}$ : temperature & 36.430 & 10.120 & $21{ }^{\circ} \mathrm{C}$ \\
$\mathrm{S}_{2}$ : humidity & 36.000 & 10.147 & $65 \%$ \\
$\mathrm{~S}_{3}$ : sunshine & 36.920 & 10.650 & $\mathbf{6 0 0} \mathrm{w} / \mathrm{m}^{2}$ \\
$\mathrm{~S}_{4}$ : wind speed & 36.500 & 10.710 & $12 \mathrm{Km} / \mathrm{h}$ \\
\hline \hline
\end{tabular}

Table4 show the recording of the attribute and the spatial data during the detection of position change and the value measured by the sensor $\mathrm{S} 4$ between $\mathrm{T} 2$ and $\mathrm{T} 3$
TABLE 4: Value and position of S4 has been changed

\begin{tabular}{lccc}
\hline \multicolumn{1}{c}{ Sensor } & latitude & Longitude & value \\
\hline $\mathrm{S}_{1}$ : temperature & 36.340 & 10.120 & $21^{\circ} \mathrm{C}$ \\
$\mathrm{S}_{2}$ : humidity & 36.000 & 10.147 & $65 \%$ \\
$\mathrm{~S}_{3}$ : sunshine & 36.920 & 10.650 & $600 \mathrm{w} / \mathrm{m}^{2}$ \\
$\mathrm{~S}_{4}$ : wind speed & $\mathbf{3 6 . 1 0 0}$ & $\mathbf{1 0 . 0 9 0}$ & $\mathbf{2 0 ~ K m} / \mathrm{h}$ \\
\hline \hline
\end{tabular}

Table 5 shows changes in positions and values measured between T3 and T4 for the two sensors S2 and S3. And shows a change in value level for sensor $\mathrm{S} 1$.

TABLE. 5: The change between T3 and T4

\begin{tabular}{lllc}
\hline \hline Sensor & latitude & Longitude & value \\
\hline S1: Temperature & 36.340 & 10.120 & $\mathbf{2 2}{ }^{\circ} \mathbf{C}$ \\
$\mathrm{S}_{2}$ : Humidity & $\mathbf{3 6 . 0 1 0}$ & $\mathbf{1 0 . 1 5 2}$ & $\mathbf{6 2 \%}$ \\
$\mathrm{S}_{3}$ : Sunshine & $\mathbf{3 5 . 9 2 0}$ & $\mathbf{1 1 . 6 5 0}$ & $\mathbf{5 0 0} \mathrm{w} / \mathrm{m}^{2}$ \\
$\mathrm{~S}_{4}$ : Wind speed & 36.100 & 10.090 & $20 \mathrm{Km} / \mathrm{h}$ \\
\hline \hline
\end{tabular}

\section{SPATIO-TEMPORAL DATA MODEL}

\subsection{Real-Time Spatio-Temporal Conceptual Model}

There are various spatial Conceptual Models of Data (CMD) among which we state two different approaches: Perceptory [8] and Modeling of Application Data with Spatio-Temporal (MADS) [9][10]. They are based on CMD that originates from the databases, respectively UML and ER (Entity/Association). They are extended to the spatial concepts.

Considering the limitations related to MADS [11], we chose to use the Preceptory tool. This tool, which can be integrated into the Engineering Software Workshop (ESW) Visio, offers its effectiveness in the visual modeling of Spatial Data Bases (DBS) and the basics of Spatio-Temporal data [8]. The Perception approach was developed starting from a standard Directed-Object formalism. Later, it was developed and enriched to support the spatial reference considering the ISO-TC211 norms.

The model mainly describes the aspects of environmental monitoring. In this model of RealTime Spatio-Temporal Data (RTSTD), we define a GeoUML representation of the Spatio-Temporal data in real-time with the Perceptory tool [12] as well as their relationship with other static entities. The data collected by a sensor node are called 
according to their locations and their dates of acquisition

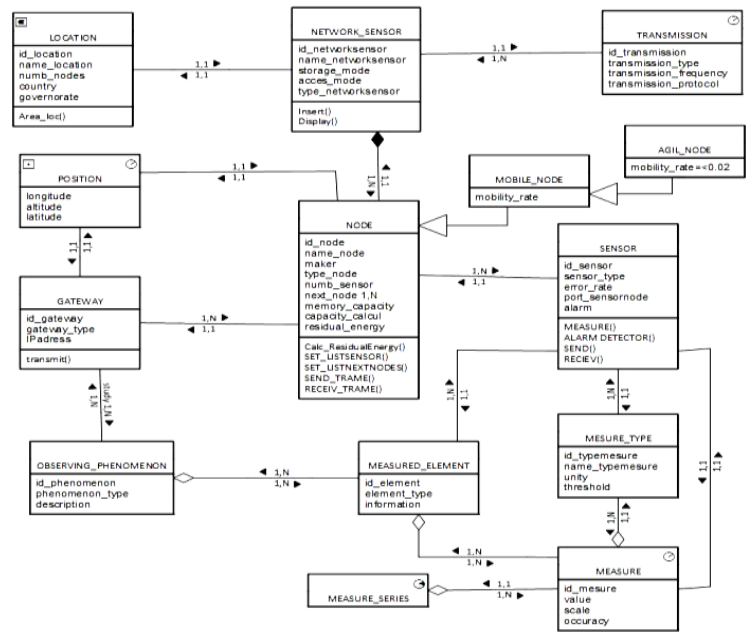

FIG.2. Real-Time Spatio-Temporal Conceptual Model

\subsection{Model Description}

The model described in Fig. 2 represents a realtime Spatio-Temporal conceptual model by GeoUML. The main elements of the model presented in the previous Figure. describe the aspects of the monitoring application in the environmental field.

A sensors network is in a study area; it is composed of a set of sensor nodes. These sensor nodes can be communicated by a set of microsensors located in the same observation medium "zone", with a fixed or a variable position (Temperature, humidity, pressure) at different positions (mobile and fixed sensors). Each one of these sensors has a measurement type (Measurement Unit) which data is obtained from measurements.

The gateway is responsible for monitoring the various phenomena (Pollution, drought, Volcanoes) and transmitting the measurements collected by the sensor nodes. These different phenomena are analyzed from one or several elements to measure the evolution of the phenomena. For the time and space measurements (the case of mobile sensors), it is possible to acquire or correct the measurements variations. The dynamic objects during these measurement campaigns concern the objects changing value, shape, or position in time and/or space.

\section{QUERIES PROCESSING ON DATA MODEL}

The data acquired by the sensors are localized and dated. They are used in real-time and deferred time managing a variety of large masses of SpatioTemporal data. Our data model allows executing several types of queries [8]: spatial queries, temporal and Spatio-Temporal. We can mention examples of requests processed by our system:

- Spatial Queries: They allow selecting the detected point at a given zone or geographical position. For example, the query displays the different measurements collected by the sensors located in an area selected by the user.

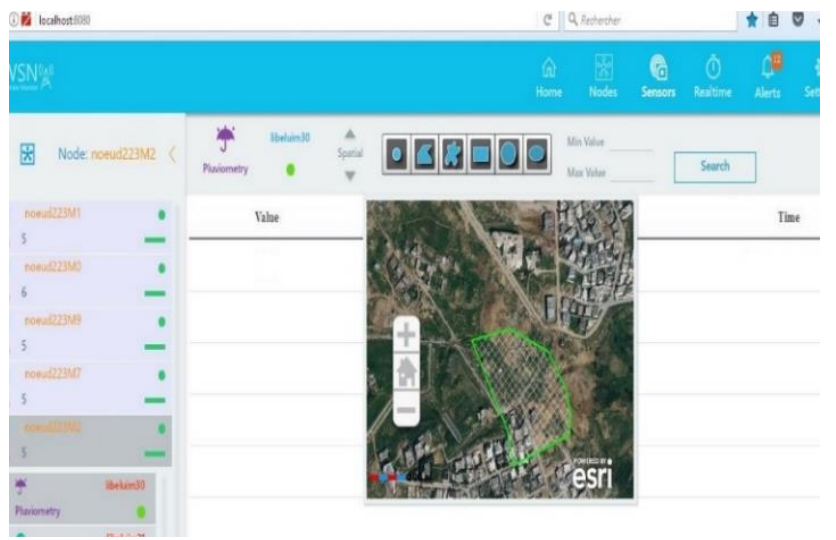

FIG.3. Spatial Queries Processing

Fig. 3 shows a spatial functionality, specifying the sensors localized in the selected area and showing the various measures taken.

- Time Queries: They are used to select the detected values at a time or a time interval.

For example, the query: "What are the rainfall amounts for the period [T1, T2]?"

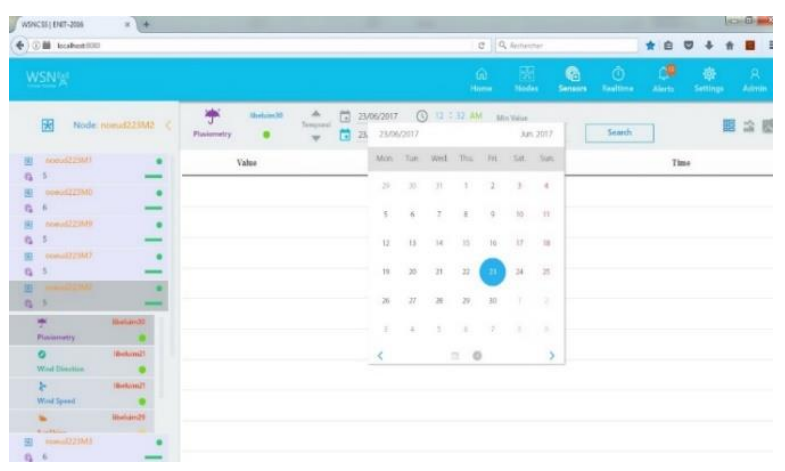

FIG.4. Time Queries Processing 
Fig. 4 represents the case in which the users retrieve the observations obtained in the period from a start time to an end time according to their choice.

- Spatio-temporal queries: Spatio-temporal queries have different types and they classify data types in three categories [2]: Where, When, and What. Hence, any spatiotemporal request can be categorized as one of the types of requests below. $\checkmark \quad$ For a given location and during the period [T1, T2], we collect different measures close to the location.

When+ Where $\rightarrow$

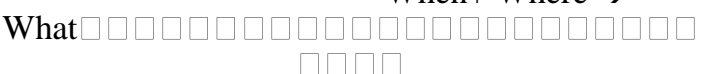

For example : "Select Position, Sensor, Node, Mesure, Time from measures_table, measures_element, positions, sensors, nodes where (id_position = node.id_position) and (id_node = sensor.id_node) and (id_sensor $=$ measure.id_sensor) and (element_type = 'Temperature') and (ST_distance (ST_AsText (geom), 'POINT $(10.146637 .8306) ')=($ select min $($ ST_distance (ST_AsText (geom), 'POINT(10.1466 37.8 306)')) from position)) and measure_time between '2017-01 -06 19:30:50.67' AND'2017-01 -06 20: 28: 30 .67' ORDER BY measures_time ASC "

This request uses the spatial functions that specifies which sensors are nearest to the actual location at a given moment and displays the several measurements that have been collected. For a specific time and a specific value of temperature, which is the sensor position?

When+ What $\rightarrow$ Where

(2)

For example, "Select ST_AsText (geom), from Nodes, Positions, Measures, measures_element, sensors where id_position $=$ node.id_position and id_node = sensor.id_node and id_sensor = measure.id_sensor and element_type = 'Temperature' and measure_time $=$ '2015-03-25 $12: 41: 23$ 'and value $=39 "$.

This query determines the position (or positions) for a temperature value equal to 39 at a given instant.

For a given location what are the measurement times in which the temperature is equal to 39 ?

Where+ What $\rightarrow$ When

For example, "select mesure_time from node, position, measure, measure_element, sensor where id_position= node.id_position and id_node= sensor.id_node and id_sensor= measure.id_sensor and element_type $=$ 'temperature' and ST_AsText $($ geom $)=$ 'POINT(10.1466 36.8306)'and value $=39 "$.

This query determines the times (when) for a temperature value equal to 39 at the position (10.1466 36.8306).

$\checkmark$ For a given location: What are the temperature values detected and the measurement times?

Where $\rightarrow$ What + When

For example, "select value, measure_time from node, position, measure, measure_element, sensor where id_position= node.id_position and id_node= sensor.id_node and id_sensor= measure.id_sensor and element_type $=$ 'temperature' and ST_AsText(geom) = 'POINT(10.1466 36.8306)".

This request has a sensor position, making it possible to determine the different collected measurements and the correspondent times.

For a value lower than a given temperature value, find the time and location.

$$
\text { What } \rightarrow \text { Where+ When }
$$

For example, "select value, ST_AsText (Geom), measure_time from measure, measure_element, position where element_ type='temperature' and value $<=39 "$.

This query has temperature values lower than a given value, with the determination of where (the position) and when (time) these measurements were performed.

$\checkmark$ For a given instant find the value of the temperature and the sensor position which collected the measures at this time.

When $\rightarrow$ What + Where

For example, "select value, ST_AsText(Geom) from node, position, measure, measure_element, a sensor where id_position $=$ node.id_position and id_node $=$ sensor.id_node and id_sensor = measure.id_sensor and element_type = 'temperature' and measure_time = '2015-03-25 12:41:23". 
This request contains a measurement time making it possible to determine the temperature value and thus its position (or positions).

\section{RESULTS}

To determine the performance and the efficiency of our data model as well as the implementation of the Spatio-Temporal conceptual modeling by GeoUML in PostgreSQL /PostGIS time-space database, several types of queries have been executed at the database level to different forms in the previous section.

In addition, the consultation, and the manipulation of data by the open-source GIS QGIS software (Fig. 5) allow the display of data in vectorial or alphanumeric format. Likewise, for our system of climatic monitoring, we must execute spatial queries Fig.3, temporal Fig.4, and SpatioTemporal allowing thus the following of different data evolution.

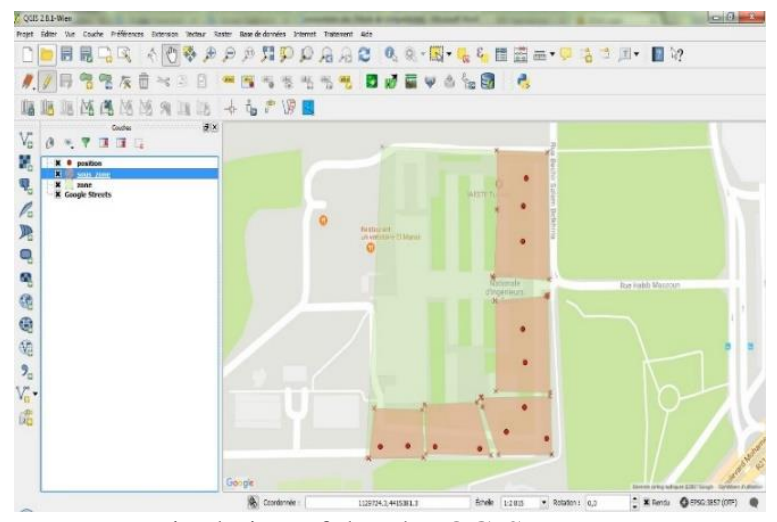

FIG.5. Manipulation of data by QGIS

\section{CONCLUSION}

In the old method of recording, for each new measurement, a new row will be added to the database table containing the redundant information. For example, to record the new temperature measurement we do not need to record the position of the sensors if it is not moved. With our model, we have subdivided the save sets into several sub entities to minimize the feedback and save only the relevant information. This is a new type of interaction between the two domains: temporal and spatial. The innovative aspect is that the model is based on a "snapshot model using object-oriented concepts" instead of the "snapshot model". With this approach, the information about the identity of the sensor object is maintained over time, allowing for periodic, instantaneous, or eventbased monitoring of space and changes. The approach also incorporates modeling of events that impact the monitoring area.

\section{ACKNOWLEDGMENT}

This work was carried out within the framework of a scientific research program of the Tunisian-South African cooperation (grants $\left.n^{\circ} 113340,120106\right)$. We would like to thank the Tunisian Ministry of Higher Education and Scientific Research for the support given to this research project.

\section{REFERENCES}

[1] Z. Jiang and S. Shekhar, "Spatial and Spatiotemporal Big Data Science," Spat. Big Data Sci., pp. 15-44, 2017, doi: 10.1007/978-3319-60195-3_2.

[2] R. Rajan, S. Profile, S. S. Rajest, B. Singh, and R. Regin, "Spatial Data Mining Methods Databases and Statistics Point of Views ," 1200, doi: 10.46532/978-81-950008-7-6_010.

[3] G. Lü, M. Batty, J. Strobl, H. Lin, A. X. Zhu, and M. Chen, "Reflections and speculations on the progress in Geographic Information Systems (GIS): a geographic perspective," https://doi.org/10.1080/13658816.2018.153313 6, vol. 33, no. 2, pp. 346-367, Feb. 2018, doi: 10.1080/13658816.2018.1533136.

[4] P. Jia, X. Cheng, H. Xue, and Y. Wang, "Applications of geographic information systems (GIS) data and methods in obesityrelated research," Obes. Rev., vol. 18, no. 4, pp. 400-411, Apr. 2017, doi: 10.1111/OBR.12495.

[5] D. Gounaridis, I. Chorianopoulos, E. Symeonakis, and S. Koukoulas, "A Random Forest-Cellular Automata modelling approach to explore future land use/cover change in Attica (Greece), under different socio-economic realities and scales," Sci. Total Environ., vol. 646, pp. 320-335, Jan. 2019, doi: 10.1016/J.SCITOTENV.2018.07.302.

[6] T. Sautory, N. Cingillioglu, and A. Russo, "HySTER: A Hybrid Spatio-Temporal Event Reasoner,” Jan. 2021, Accessed: Nov. 27, 2021. 
[Online]. Available: https://arxiv.org/abs/2101.06644v1.

[7] W. Xiong, H. Chen, N. Guo, Q. Gong, and W. Luo, "ENSTDM: An ENtity-Based SpatioTemporal Data Model and Case Study in Real Estate Management," Lect. Notes Comput. Sci. (including Subser. Lect. Notes Artif. Intell. Lect. Notes Bioinformatics), vol. 12753 LNCS, pp. 27-42, Apr. 2021, doi: 10.1007/978-3-03085462-1_3.

[8] F. Walid and T. Ezzedine, "Design of a climate monitoring system based on sensor network," 2017 13th Int. Wirel. Commun. Mob. Comput. Conf. IWCMC 2017, pp. 1791-1796, Jul. 2017, doi: 10.1109/IWCMC.2017.7986555.

[9] A. R. Hajalalaina, S. G. Andriniaina, A. R. Hajalalaina, and S. G. Andriniaina, "A Decision Support System for Spatial Analysis of Agricultural Production in Madagascar," J. Data Anal. Inf. Process., vol. 9, no. 1, pp. 1-22, Feb. 2021, doi: 10.4236/JDAIP.2021.91001.

[10]Á. Fidalgo-Blanco, M. L. Sein-Echaluce, and F. J. García-Peñalvo, "Micro Flip Teaching with Collective Intelligence," Lect. Notes Comput. Sci. (including Subser. Lect. Notes Artif. Intell. Lect. Notes Bioinformatics), vol. 10924 LNCS, pp. 400-415, Jul. 2018, doi: 10.1007/978-3-31991743-6_30.

[11]C. Folberth, A. Baklanov, J. Balkovič, R. Skalský, N. Khabarov, and M. Obersteiner, "Spatio-temporal downscaling of gridded crop model yield estimates based on machine learning," Agric. For. Meteorol., vol. 264, pp. 115, Jan. 2019, doi: 10.1016/J.AGRFORMET.2018.09.021.

[12]K. Jetlund, E. Onstein, and L. Huang, “Adapted Rules for UML Modelling of Geospatial Information for Model-Driven Implementation as OWL Ontologies," ISPRS Int. J. GeoInformation 2019, Vol. 8, Page 365, vol. 8, no. 9, p. 365, Aug. 2019, doi: 10.3390/IJGI8090365. 